\title{
Documentos
}

\section{EL DIAGNÓSTICO PRENATAL DE CARDIOPATÍAS CONGÉNITAS MEJORA EL PRONÓSTICO NEONATAL}

\author{
Lorena Quiroz V. ${ }^{1}$, Enrique Siebald C. ${ }^{2}$, Cristian Belmar J. ${ }^{2}$, Gonzalo Urcelay M. ${ }^{3}$, Jorge \\ Carvajal C. ${ }^{2} P h D$ \\ ${ }^{1}$ Departamento de Obstetricia y Ginecología, Hospital Clínico, Universidad de Chile. ${ }^{2}$ Departamento de Obstetricia y \\ Ginecología, ${ }^{3}$ Departamento de Pediatría, Facultad de Medicina, Pontificia Universidad Católica de Chile.
}

\section{RESUMEN}

Las malformaciones congénitas son la principal causa de muerte neonatal precoz en nuestro medio; en la mayoría de los casos corresponden a cardiopatías congénitas mayores. Las cardiopatías congénitas tienen una incidencia de 8/1.000 recién nacidos vivos, correspondiendo la mitad de ellas a cardiopatías congénitas mayores. Una de las intervenciones recomendadas para reducir la mortalidad de este grupo de niños es evaluar rutinariamente la anatomía del corazón fetal mediante ecografía obstétrica, para planificar la atención neonatal, de aquellos fetos con cardiopatías congénitas, en el momento y lugar más oportuno. En objetivo de la presente revisión es comprobar si el diagnóstico prenatal de una cardiopatía congénita mejora el pronóstico perinatal respecto de aquellos casos que son diagnosticados post parto. Observamos que el diagnóstico antenatal de cardiopatía congénita, no mejora la sobrevida neonatal, excepto en ciertas cardiopatías congénitas ductus dependientes (transposición de grandes arterias, hipoplasia del corazón izquierdo y coartación de aorta), en que si se ha reportado una mayor probabilidad de sobrevida en el grupo de recién nacidos en los cuales se realizó el diagnóstico en el período prenatal. Recomendamos la evaluación rutinaria del corazón fetal en la ecografía obstétrica habitual, y efectuar ecocardiografía fetal especializada ante la sospecha de alteraciones o en aquellos grupos de mayor riesgo.

\section{PALABRAS CLAVES: Cardiopatías congénitas mayores, diagnóstico prenatal, ecocardiografía fetal}

\section{SUMMARY}

Congenital malformations are the main cause of neonatal death; in most of the cases they correspond to major congenital heart defects. Congenital heart defects have an incidence of $8 / 1,000$ live newborns, corresponding half of them to major congenital heart defects. To reduce the mortality of this group of children routine evaluation of fetal heart anatomy by ultrasound is recommended, allowing neonatal care, of those fetuses with congenital heart defects, at the appropriate time and place. Here we attempt to verify if prenatal diagnosis of a congenital heart defect improves the perinatal outcome compare to postnatal diagnosis. We observed that the prenatal diagnosis of congenital heart defects, does not improve the neonatal outcome, except in certain congenital ductus dependent heart defects (transposition of great vessels, hypoplastic left heart syndrome and aortic coarctation), where a better outcome has been shown for those new born with prenatal diagnosis. We recommend routine evaluation of the fetal heart during prenatal ultrasound, and to carry out fetal echocardiography in high risk groups or when a defect is suspected.

KEY WORDS: Major congenital heart defects, prenatal diagnosis, fetal echocardiography 


\section{INTRODUCCIÓN}

La mortalidad neonatal precoz en Chile es de $4,5 / 1000 \mathrm{nv}$; el $40 \%$ de las muertes se deben a malformaciones congénitas (1). Las malformaciones congénitas son además, la segunda causa de muerte en menores de 1 año, siendo responsables del $32 \%$ de las defunciones en este grupo de edad (2). La mayoría de las muertes neonatales por malformaciones se deben a cardiopatías congénitas.

Las cardiopatías congénitas son todas las malformaciones cardíacas que están presentes en el momento del nacimiento y son secundarias a alteraciones en la organogénesis, desconociéndose en la gran mayoría de los casos los factores causales (85-90\%). Se estima una incidencia de cardiopatías congénitas de 8/1.000 nv; incidencia bastante constante a nivel mundial, independiente de factores como raza, condición socioeconómica o situación geográfica (3). Las cardiopatías congénitas mayores tienen una prevalencia estimada de 4/1.000 nv (4), entendiendo por tales a aquellas malformaciones complejas del corazón o de las grandes arterias, y/o la presencia de anomalías estructurales que requieren de una intervención quirúrgica o cateterismo dentro de los primeros 6 meses de vida (5).

La detección de anomalías congénitas mayores es uno de los objetivos específicos del examen ultrasonográfico de rutina durante el embarazo. En Chile, se ha implementado la ultrasonografía prenatal de rutina con la idea de seleccionar a la población de riesgo, la cual es derivada al nivel terciario, buscando concentrar recursos y disminuir la morbilidad y mortalidad perinatal (6). Una de las intervenciones recomendadas en el plan AUGE respecto a cardiopatías congénitas es realizar screening mediante ecografía obstétrica para detectar cardiopatías congénitas y, en especial, aquellas cardiopatías complejas que llevan a un rápido deterioro clínico del neonato luego del nacimiento (drenaje venoso anómalo pulmonar total y lesiones obstructivas izquierdas). En una siguiente etapa, en los centros de referencia, estas pacientes son evaluadas por especialistas calificados, con medios técnicos apropiados; estableciendo continuidad con el manejo postnatal (5).

La aplicación del ultrasonido de rutina y su impacto en la reducción de la morbimortalidad perinatal ha sido tema de debate. En la Tabla I se resumen algunos de los principales estudios al respecto. Entre ellos se destacan dos estudios que analizan el problema con conclusiones discre- pantes: The Helsinki Ultrasound Trial (1990) (7) y The Routine Antenatal Diagnostic Imaging with Ultrasound (RADIUS; 1993) (8).

The Helsinki Ultrasound Trial (7), efectuado en dos centros en Finlandia, randomizó 4.691 pacientes embarazadas. A un grupo se realizó un ultrasonido de rutina entre las 16 y 20 semanas de gestación y al otro sólo se realizó una evaluación ecográfica según sospecha médica del obstetra tratante (grupo control). La mortalidad perinatal fue significativamente menor en el grupo screening que en el grupo control (4,6/1000 v/s 9,0/1000), sin embargo es muy probable que esta reducción $(49,2 \%)$ en la mortalidad se haya debido a la mayor tasa de abortos provocados por la detección precoz de malformaciones congénitas mayores. La sensibilidad reportada en la detección de malformaciones en el grupo de screening fue de $36 \%$.

En RADIUS (8), se randomizaron $15.151 \mathrm{em}-$ barazadas de bajo riesgo; al grupo de estudio se realizaron dos ecografías de rutina (entre las 15 a 22 semanas de gestación y entre las 31 y 35 semanas), al grupo control se realizó evaluación ultrasonográfica según indicación médica del obstetra tratante. El número promedio de ecografías fue de 2,2 en el grupo de estudio y 0,6 en el grupo control. La tasa de resultados perinatales adversos (muerte fetal y neonatal y morbilidad neonatal) no tuvo diferencia significativa entre los grupos ( $5 \%$ v/s 4,9\%; RR 1,0; IC95\% 0,9-1,2; $\mathrm{p}=0,85)$. Se concluyó que la detección ultrasonográfica de malformaciones congénitas no afectó el resultado perinatal. Sin embargo, una de las mayores críticas a este estudio fue la baja tasa de malformaciones reportada $(3,6 / 1.000)$, por lo que el impacto del examen pudo haber sido subestimado.

Considerando la importancia de las cardiopatías congénitas como causa de muerte en el período perinatal, y las implicancias posibles de su diagnóstico antenatal, al adecuar el manejo materno fetal mediante la derivación oportuna a un centro terciario para el control del embarazo, resolución del parto y atención oportuna del recién nacido; se ha postulado que el diagnóstico antenatal de las cardiopatías congénitas permitiría reducir la mortalidad perinatal de los recién nacidos con cardiopatías congénitas.

El objetivo de nuestra revisión es evaluar la información disponible, para comprobar la veracidad de esta hipótesis, es decir verificar si el diagnóstico prenatal de una cardiopatía congénita mejora el pronóstico perinatal respecto de aquellos casos que son diagnosticados en el período post parto. 


\section{ECOCARDIOGRAFIA FETAL}

La ecocardiografía fetal, que se inició a fines de los años 70 , es un procedimiento diagnóstico ultrasonográfico de tercer nivel realizado por un profesional entrenado con equipos de alta tecnología (diferentes ejes cardíacos, uso de Doppler, uso de color, etc.). Sin embargo, se refiere también como ecocardiografía al examen ultrasonográfico de nivel primario o secundario en el cual se realiza una evaluación del corazón fetal con menos detalle (visión de cuatro cámaras y tractos de salida). Esta doble utilización del mismo nombre lleva muchas veces a confusión cuando se intenta analizar las características de la prueba.

Numerosas series evalúan las tasas de sensibilidad y especificidad en diagnóstico prenatal de malformaciones cardiacas de este test diagnóstico (9-23). Los valores de especificidad reportados son bastante uniformes y por lo general mayores al $95 \%$. Sin embargo, las tasas de sensibilidad son muy variadas. Esto último se explica probablemente por las diferencias en el diseño de los trabajos, grupo de estudio (alto o bajo riesgo), tipo de equipo médico o técnico que realiza el examen (ecografía nivel l, obstetras entrenados en ecografía 3 nivel, en ecocardiografía fetal, cardiólogos pediátricos, etc.), diferentes edades gestacionales, etc. La tasa de detección de defectos ventriculoseptales (con una incidencia conocida de 1 en 200 a 1 en 400) ha sido propuesta como un buen marcador de la sensibilidad del examen en un estudio dado. El rango reportado de sensibilidad varía desde $2,6 \%$ a $92 \%$. Debemos señalar que esta alta tasa de sensibilidad del $92 \%$, especificidad del $99,7 \%$, VPP del $95,8 \%$ y VPN del $99,4 \%$ con la visión de cuatro cámaras fue reportada en un estudio en donde se concentró pacientes de alto riesgo y las ecocardiografías fueron realizadas por ecografistas experimentados (9).

Tradicionalmente, se ha propuesto reservar el estudio ecocardiográfico a pacientes de alto riesgo, sin embargo, en la generalidad de los reportes que evalúan población de alto y bajo riesgo, se concluye que la mayoría de los fetos con diagnóstico de cardiopatía no presentan factor de riesgo alguno $(10,14,19)$ y entre 20 y $50 \%$ de los casos son pacientes derivadas por sospecha de cardiopatía en ecografía de screening (población general) $(9,21)$, lo que permite suponer que destinar el test sólo al grupo de alto riesgo resultaría en pérdida del mayor número de casos.

Para mejorar la detección en ecografías de screenig se ha propuesto el uso de la visión de 4 cámaras y la visión de tractos de salida $(10,12)$; esta última permite una mayor sensibilidad en el diagnóstico de patologías tales como Tetralogía de Fallot y Transposición de grandes vasos (malformaciones cardiacas mayores más frecuentes junto a hipoplasia ventrículo izquierdo y canal AV). La visión de 4 cámaras se ha relacionado además con un alto valor predictivo negativo $(99,4 \%)$ (9). Un estudio prospectivo de cohorte concluyó, sin embargo, que la visión de 4 cámaras y tractos de salida realizada en una población de bajo riesgo tenía una sensibilidad muy por debajo que aquella reportada por la ecocardiografía "detallada" en una población de alto riesgo (14,3\% v/s 62,5\%) (14).

\section{DIAGNÓSTICO PRENATAL Y PRONÓSTICO NEONATAL}

Se ha propuesto que el diagnóstico prenatal de cardiopatías congénitas complejas puede modificar el pronóstico neonatal al planificar el parto en un centro especializado y preparado para recibir un recién nacido con estas características. Algunas medidas como la infusión precoz de prostaglandinas o la septostomía atrial con balón, de urgencia, podrían mejorar el pronóstico en aquellos casos en que se requiere mantener los cortocircuitos de flujo circulatorio fetal, como por ejemplo la Trans-

Tabla I

SENSIBILIDAD Y ESPECIFICIDAD DEL ULTRASONIDO PARA EL DIAGNÓSTICO DE MALFORMACIONES CONGÉNITAS*

\begin{tabular}{lrccr}
\hline Estudio & $n$ & Prevalencia (\%) & Sensibilidad (\%) & Especificidad (\%) \\
\hline Helsinski (7) & 4.691 & 0,99 & 36,0 & 99,8 \\
Bélgica (49) & 16.221 & 1,61 & 20,8 & 100,0 \\
RADIUS (8) & 7.475 & 2,40 & 16,6 & 99,9 \\
Eurofetus (48) & 200.000 & 2,0 & 70,0 & 99,0 \\
\hline
\end{tabular}

*Se observa una especificidad elevada y constante, mientras que la sensibilidad es muy variable. Las diferencias se explican probablemente pues los estudios son diferentes en cuento a la población estudiada y al tipo de médicos involucrados en el examen. 
posición de Grandes Vasos o la Hipoplasia de Ventrículo Izquierdo (24), sin embargo, no existe consenso y ha sido difícil de demostrar la utilidad real de la detección prenatal de la cardiopatía, como un medio eficaz para mejorar el pronóstico neonatal (5).

Lograr clarificar completamente esta disyuntiva enfrenta dificultades inherentes a la patología y a su asociación con otras enfermedades. Las cardiopatías que son detectadas en la vida fetal, en la mayoría de los casos corresponden a malformaciones congénitas severas (23). Además muchas cardiopatías se asocian a aneuploidias $(9,13,24)$ y a otras malformaciones extracardiacas (25), lo cual además empeora el pronóstico de la cardiopatía y aumenta el riesgo de muerte fetal y neonatal $(26,27)$. Estos problemas asociados explican por ejemplo la gran variabilidad reportada en la sobrevida neonatal, con tasas entre el 38 y $77 \%$ (29-32). Otra fuente de confusión es la interrupción electiva del embarazo en algunos países donde el aborto es legal y culturalmente aceptable, dado el mal pronóstico perinatal (33).

La evaluación observacional no mostró diferencias significativas en las tasas de sobrevida en aquellas pacientes en que el diagnóstico de cardiopatía se efectuó de modo antenatal, comparado con aquellos en que el diagnóstico se hizo en el período neonatal $(77 \% \mathrm{v} / \mathrm{s} 66 \%)$. Sin embargo, el grupo de pacientes a quienes se realizó el diagnóstico prenatalmente presentaron con menor frecuencia acidosis metabólica (6\% v/s $45 \%)$ y menos episodios de paro cardiaco $(0 \% \mathrm{v} / \mathrm{s} 9 \%)$ (32-35).

Un estudio de cohorte comparó dos grupos con diagnóstico de cardiopatía congénita significativa como malformación única; una cohorte correspondía a quienes se les había realizado el diagnóstico en el período prenatal (45 casos) y la otra cohorte a quienes el diagnóstico se había realizado en el período postnatal (54 casos). Se observó que el $80 \%$ de los casos con diagnóstico prenatal sobrevivieron (alta a domicilio) v/s $67 \%$ de los casos diagnosticados en el período postnatal $(p=0,14)$. El análisis de los datos no demostró diferencia en los casos de cardiopatías ductus-dependiente ni en los casos de hipoplasia ventrículo izquierdo. Sólo se demostró mejor pronóstico en aquellos casos en donde fue posible realizar una reparación quirúrgica biventricular (96\% v/s 76\%, p<0,05), mientras que en los casos que la reparación quirúrgica obtuvo un único ventrículo funcional no se evidenció una diferencia significativa (36).

Del mismo modo, estudios que evalúan pará- metros fisiológicos (28) y bioquímicos (37) no lograron demostrar el beneficio del diagnóstico prenatal de la cardiopatía. En la Tabla II se resumen las tasas de mortalidad de aquellos estudios que comparan diagnóstico prenatal $\mathrm{v} / \mathrm{s}$ postnatal de cardiopatías congénitas.

De estos datos se concluye que no existe una reducción en la mortalidad neonatal al realizar el diagnóstico prenatal de cardiopatías congénitas $(19,6 \% \mathrm{v} / \mathrm{s} 18,9 \%$ diferencia no significativa). Estos datos parecen desalentadores (38), sin embargo, como se mostrará a continuación, la evaluación es más optimista cuando se analizan grupos especiales de cardiopatías congénitas, existiendo evidencias para sostener que el diagnóstico prenatal beneficia a los neonatos portadores de ciertas patologías específicas, ductus dependientes, tales como: transposición de los grandes vasos (39), hipoplasia del corazón izquierdo (43) y coartación aórtica crítica (44).

Transposición de grandes vasos. Es una cardiopatía congénita en la cual se produce un deterioro progresivo en la oxigenación que puede conducir a la muerte del recién nacido en sus primeras horas de vida, debido a los cambios fisiológicos propios de la adaptación neonatal. La septostomía atrial de emergencia y la infusión precoz de prostaglandinas son requeridas para permitir la sobrevida de estos pacientes. En estos casos las anomalías extra cardiacas son infrecuentes (45) y la sobrevida postoperatoria es mayor del

Tabla II

\section{COMPARACIÓN DE LA MORTALIDAD POR CARDIOPATÍAS CONGÉNITAS SEGÚN MOMENTO DEL DIAGNÓSTICO*}

\begin{tabular}{lll}
\hline Estudio & Prenatal (\%) & Postnatal (\%) \\
\hline Kern, 1997 & $4 / 15(26,7)$ & $7 / 38(18,5)$ \\
Copel, 1997 & $9 / 45(20,0)$ & $18 / 54(33,0)$ \\
Bonnet, 1999 & $0 / 68(0)$ & $35 / 250(14,0)$ \\
Kumar, 1999 & $13 / 41(31,7)$ & $21 / 75(28,0)$ \\
Simpson, 2000 & $9 / 65(13,9)$ & $14 / 95(14,8)$ \\
Mahle, 2001 & $29 / 79(36,7)$ & $38 / 137(27,8)$ \\
Jaeggi, 2001 & $23 / 99(23,0)$ & $84 / 562(15,0)$ \\
Tworetzky, 2001 & $0 / 22(0)$ & $13 / 38(34,0)$ \\
Franklin, 2002 & $0 / 10(0)$ & $10 / 22(45,6)$ \\
\hline Total & $87 / 444(19,6)$ & $240 / 1271(18,9)$ \\
\hline
\end{tabular}

*Estos estudios no demuestran una reducción en el riesgo de muerte en el período neonatal si el diagnóstico de cardiopatía congénita se efectuó de modo antenatal. En la tabla se presentan los resultados como número de muertes sobre el total de niños diagnosticados y en paréntesis el porcentaje. 
$95 \%(40,45)$, por lo tanto, se ha postulado que este grupo de pacientes tendría un mayor beneficio al ser atendidos en un centro especializado al momento de nacer. De hecho la demora en el traslado al centro terciario fue identificada como uno de los factores de riesgo de muerte preoperatorio en este grupo (45)

Un análisis retrospectivo comparó pacientes con transposición de grandes vasos; 68 recién nacidos con diagnóstico prenatal y 250 con diagnóstico postnatal. El tiempo promedio entre el nacimiento y la admisión en una unidad especializada fue de 2 horas en el grupo prenatal y de 73 horas en el grupo postnatal $(p<0,01)$. Hubo más casos de acidosis metabólica y falla multiorgánica en el grupo con diagnóstico postnatal $(p<0,01)$. La mortalidad preoperatoria $(6 \% \mathrm{v} / \mathrm{s} 0 \% ; p<0,05)$ y postoperatoria $(8,5 \% \mathrm{v} / \mathrm{s} 0 \% ; \mathrm{p}<0,01)$ fue significativamente mayor en el grupo con diagnóstico postnatal. La septostomía atrial de urgencia fue necesaria en un $12 \%$ del grupo con diagnóstico prenatal (32).

Hipoplasia de ventrículo izquierdo. El síndrome de hipoplasia de ventrículo izquierdo representa un caso especialmente complejo, puesto que el recién nacido parece saludable al momento de nacer, sin embargo, evoluciona con un rápido deterioro hemodinámico al cerrarse el ductus arterioso (46). La mortalidad postoperatoria es de un $40-50 \%$ aproximadamente $(31,38)$ y los trastornos neurocognitivos son frecuentes. El parto en un centro neonatal especializado permitiría la administración precoz de prostaglandinas reduciendo el riesgo de hipoxia y muerte (33).

Aunque ha sido reportado el beneficio de reducir la mortalidad al realizar la cirugía de Norwood, cuando el diagnóstico se realizó en el período prenatal $(0 \% \mathrm{v} / \mathrm{s} 34 \%)(42)$, no se ha demostrado de modo consistente una reducción en la mortalidad neonatal en asociación con el diagnóstico antenatal de hipoplasia del VI $(38,43)$. Sin embargo, el grupo de diagnóstico prenatal presentó menos eventos neurológicos adversos $(15,2 \% \mathrm{v} / \mathrm{s}$. 27,7\%; OR; 0,43: 0,18-1,0) (43), y una mejor condición metabólica pre operatoria $(40,42)$.

Coartación aórtica. La coartación aórtica de presentación neonatal (forma infantil) es un cuadro congénito ductus-dependiente, de esta forma, evoluciona con frecuencia hacia el colapso circulatorio al cerrarse el ductus en el periodo postnatal. Se ha reportado que el diagnóstico postnatal de esta cardiopatía se asocia con más casos de deterioro hemodinámico severo (36), colapso circulatorio y muerte $(45,5 \% \mathrm{v} / \mathrm{s} 0 \% ; \mathrm{p}<0,02)(42)$.

\section{CONCLUSIONES}

Las malformaciones congénitas son la principal causa de muerte neonatal precoz en nuestro medio y en la mayoría de los casos corresponden a cardiopatías congénitas mayores. Con el progreso de la ultrasonografía fetal es posible diagnosticar estas anomalías antes del nacimiento, permitiendo la planificación del parto en un centro terciario, con la infraestructura y personal apropiado para atender este tipo de pacientes. Esto tiene especial relevancia en Chile, considerando las características de nuestra geografía, en que los centros de referencia se concentran en la región metropolitana y en que la derivación postnatal es difícil y muchas veces más lenta que lo deseable.

Numerosos estudios observacionales comparan el resultado perinatal de neonatos portadores de cardiopatías congénitas según el momento en el cual se realiza el diagnóstico (período antenatal o post natal). Estos estudios no demuestran una mejoría en la sobrevida neonatal secundaria al diagnóstico prenatal. Sin embargo, debe destacarse que la mayoría de los reportes corresponden a partos atendidos en centros de alta complejidad con facilidades para derivar, lo cual eventualmente habría evitado la demora en el manejo de los neonatos afectados sin diagnóstico prenatal.

A pesar de ello, evidenciamos que en ciertas cardiopatías congénitas, principalmente aquellas ductus dependientes, tales como: transposición de grandes arterias, hipoplasia del corazón izquierdo y coartación de aorta, si se ha reportado una mayor probabilidad de sobrevida en el grupo de recién nacidos en los cuales se realizó el diagnóstico en el período prenatal. Esta mejoría muy probablemente es secundaria a la necesidad de instauración de un tratamiento precoz, ya sea para mejorar la oxigenación o para mantener una hemodinamia adecuada.

En resumen, no se ha demostrado el beneficio del diagnóstico antenatal de cardiopatías congénitas, excepto para algunas condiciones específicas. Es posible que la derivación oportuna de los neonatos sea también una medida efectiva de manejo de este problema. Estimamos que en Chile, donde no existe la posibilidad de atención inmediata de recién nacidos cardiópatas, o su derivación inmediata a centros de alta complejidad, es deseable que el parto se efectúe en centros terciarios, lo cual es posible de lograr mediante una adecuada evaluación de la anatomía cardiaca fetal, en el control ecográfico de rutina.

En el análisis de esta problemática debemos 
considerar las siguientes limitaciones: 1. la mayoría de las cardiopatías congénitas se presentan en población sin factores de riesgo; 2 . no hay marcadores o factores de riesgo que permitan detectar sólo aquellas cardiopatías congénitas que se beneficiarían de diagnóstico prenatal; 3. nuestra realidad geográfica y organizativa no refleja necesariamente el escenario en que se ha sugerido la falta de utilidad del diagnóstico prenatal

Recomendamos la evaluación cardiofetal en todas las pacientes sometidas a ecografía de rutina, y su derivación para ecocardiografía de mayor complejidad en los casos de sospecha ecográfica, o en aquellos con factores de riesgo.

La pauta de manejo en la unidad de Medicina Materno Fetal de la Pontificia Universidad Católica es:

1. Realizar evaluación de la anatomía cardiaca en todas las pacientes sometidas a ultrasonido de rutina a fines del segundo trimestre.

2. Realizar capacitación permanente a los miembros del equipo médico que realizan ultrasonido, puesto que esto ha demostrado ser determinante en la sensibilidad del examen.

3. Realizar evaluación lo más completa posible de acuerdo a la capacidad del operador, pero incluyendo al menos visualización de las cuatro cámaras cardiacas y tractos de salida.

4. Referir a una unidad especializada a todos los casos con sospecha o anomalía confirmada para optimizar su manejo y resolución perinatal, en especial aquellas cardiopatías ductus dependiente.

Pensamos que este esquema es de utilidad en mejorar el diagnóstico, lograr derivación oportuna, y ofrecer mejores posibilidades de sobrevida a los RN portadores de una malformación cardiaca.

\section{BIBLIOGRAFÍA}

1. Ministerio de Salud Pública de Chile. Estadística de natalidad y mortalidad, 2003.

2. Ministerio de Salud Pública de Chile. DEIS, 2005

3. Ministerio de Salud Pública de Chile. Guía clínica de cardiopatías congénitas operables en menores de 15 años. Primera edición. Santiago, 2005.

4. Hoffman J, Christianson R. Congenital heart disease in a cohort of 19,502 births with long term follow up. Am J Cardiology 1978;42:641-7.

5. Jaeggi F, Scholler G, Jones O, Cooper S. Comparative analysis of pattern, management and outcome of pre-versus postanatally diagnosed major congenital heart disease: a population.based study. Ultrasound Obstet Gynecol 2001;17:380-5.

6. Ministerio de Salud Pública de Chile. Guía perinatal. Cedip, 2002.
7. Saari-Kemppainen A, Karjalainen O, Ylostalo $P$, Heinonen OP. Ultrasound screening and perinatal mortality: controlled trial of systematic one-stage screening in pregnancy. The Helsinki Ultrasound Trial. Lancet 1990;336:387-91.

8. Ewigman B, Crane J, Frigoletto F, LeFevre M, Bain R, McNellis D and the RADIUS Study Group. Effect of prenatal ultrasound screening on perinatal outcome. New Eng J Med 1993;329:821-7.

9. Copel J, Pilu G, Green J, Hobbins J, Kleinman C. Fetal echocardiographic screening for congenital heart disease: The importance of the four-chamber view. Am J Obstet Gynecol 1987;157:648-55.

10. Bromley B, Estroff J, Sanders S, et al. Fetal echocardiography: Accuracy and limitations in a population at high and low risk for heart defects. Am J Obstet Gynecol 1992;166:1473-81.

11. Achiron R, Glaser J, Gelernter I, Hegesh J, Yagel S. Extended fetal echocardiographic examination for detecting cardiac malformations in low risk pregnancies. Br Med J 1992;304:671-4.

12. Kirk J, Riggs T, Comstock C, Lee W, Yang S, Weinhouse E. Prenatal screening for cardiac anomalies: the value of routine addition of the aortic root to the four-chamber view. Obstet Gynecol 1994;84:427-31.

13. Allan L, Sharland GK, Milburn A, et al. Prospective diagnosis of 1006 consecutive cases of congenital heart disease in the fetus. J Am Coll Cardiology 1994;23:1452-8.

14. Ott W. The accuracy of antenatal fetal echocardiography screening in high and low risk patients. Am J Obstet Gynecol 1995;172:1741-9.

15. Montaña E, Khoury M, Cragan J, Sharma S, Dhar P, Fyfe D. Trends and outcomes after prenatal diagnosis of congenital cardiac malformations by fetal echocardiography in a well defined birth population, Atlanta, Georgia, 1990-1994. J Am Coll Cardiology 1996;28:1805-9.

16. Buskens E, Grobbee D, Frohn-Mulder I, et al. Efficacy of routine fetal ultrasound screening for congenital heart disease in normal pregnancy. Circulation 1996;94:67-72.

17. Buskens E, Stewart P, Hess J, Grobbee D, Wladimiroff JW. Efficacy of fetal echocardiography and yield by risk category. Obstet Gynecol 1996;87: 423-8.

18. Skupski D, Newman S, Edersheim T, et al. The impact of routine obstetric ultrasonographic screening in a low-risk population. Am J Obstet Gynecol 1996;175:1142-5.

19. Stumpflen I, Stumpflen A, Wimmer M, Bernaschek G. Effect of detailed fetal echocardiography as part of routine prenatal ultrasonographic screening on detection of congenital heart disease. Lancet 1996; 348:854-57.

20. Bull C. On behalf of the British Paediatric Cardiac Association. Current and potential impact of fetal diagnosis on the prevalence and spectrum of serious 
congenital heart disease at term. Lancet 1999;354: 1242-7.

21. Yates RWM. Cardiac abnormalities and their association with aneuploidy. Prenatal Diagnosis 1999;19:542-545

22. Andrews RE, Simpson JM, Sharland GK, et al. Outcome following pre-term delivery of infants antenatally diagnosed with congenital heart disease. J Pediat 2006;148(2):213-6.

23. Hoffman JIE. Incidence of congenital heart disease II. Prenatal incidence. Pediatric Cardiol 1995;16:155 65.

24. Gerlis LM. Cardiac malformations in spontaneous abortions. Int J Cardiol 1985;7:29-43.

25. Garne E, Eurocat Working Group. Prenatal diagnosis of six major cardiac malformations in Europe. A population based study. Acta Obstet Gynecol Scand 2001:80:224-8.

26. Carvalho J, Mavrides E, Shinebourne E, Campbell S, Thilaganathan B. Improving the effectiveness of routine prenatal screening for major congenital hearts defects. Heart 2002; 88: 387-91.

27. Allan LD, Hornberger L, Sharland G. Textbook of Fetal Cardiology, Greenwich Medical Media Limited: London, 2000.

28. Daubeney P, Sharland G, Cook A, Keeton B, Anderson R, Webber S. Pulmonary atresia with intact ventricular septum. Impact of fetal echocardiography on incidence at birth and postnatal outcome. Circulation 1998;98:562-66.

29. Allan LD, Huggon IC. Counselling following a diagnosis of congenital heart disease. Prenatal Diagnosis $2004 ; 24: 1136-42$

30. Tometzki A, Suda K, Kohl T, Kovalchin J, Silverman $\mathrm{N}$. Accuracy of prenatal echocardiographic diagnosis and prognosis of fetuses with conotruncal anomalies. J Am Coll Cardiology 1999;33:1696701.

31. Simpson L, Harvey-Wilkes K, D'Alton M. Congenital heart disease: the impact of delivery in a tertiary care center on SNAP score (scores for neonatal acute physiology). Am J Obstet Gynecol 2000;182(1 Pt 1):184-91.

32. Smythe J, Copel J, Kleinman C. Outcome of prenatally detected cardiac malformations. Am J Cardiology 1992;69:1471-74.

33. Allan L, Apfel H, Printz B. Outcome after prenatal diagnosis of the hypoplastic left heart syndrome. Heart 1998;79:371-73.

34. Chang A, Huhta J, Yoon G. Diagnosis, transport, and outcome in fetuses with left ventricular outflow tract obstruction. J Thor Cardiovascular Surg 1991; 102:841-8.

35. Barber G, Chin A, Murphy J, Pigott J, Norwood W. Hypoplastic left heart syndrome; lack of correlation between preoperative, demographic and laboratory findings and survival following palliative surgery. Pediatric Cardiology 1989;10:129-34.

36. Murdison K, Baffa J, Farrell P Jr, et al. Hypoplastic left heart syndrome; outcome following initial palliation. Circulation 1989;80(pt 2):IV70.

37. Copel J, Tan A, Kleinman C. Does a prenatal diagnosis of congenital heart disease alter short-term outcome? Ultrasound Obstet Gynecol 1997;10:23741.

38. Yates R. The influence of prenatal diagnosis on postnatal outcome in patients with structural congenital heart disease. Prenatal Diagnosis 2004;24: 1143-9.

39. Verheijen P, Lisowski L, Stoutenbeek P, Hitchcock J, Bennink G, Meijboom E. Lactacidosis in the neonate is minimized by prenatal detection of congenital heart disease. Ultrasound Obstet Gynecol 2002;19: 552-55.

40. Kern J, Hayes CJ, Michler RE, Gersony WM, Quaegebeur JM. Survival and risk factor analysis for the Norwood procedure for hypoplastic left heart syndrome. Am J Cardiology 1997;80:170-4.

41. Bonnet D, Coltri A, Butera G, et al. Detection of transposition of the great arteries in fetuses reduces neonatal morbidity and mortality. Circulation 1999; 99:916-18.

42. Kumar R, Newburger J, Gauvreau K, Kamenir S, Hornberger L. Comparison of outcome when hypoplastic left heart syndrome and transposition of the great arteries are diagnosed prenatally versus when diagnosis of these two conditions is made only postnatally. Am J Cardiology 1999;83:1649-53.

43. Mahle W, Clancy RR, McGaurn SP, Goin JE, Clark BJ. Impact of prenatal diagnosis on survival and early neurologic morbidity in neonates with the hypoplastic left heart syndrome. Pediatrics 2001;107: 1277-82.

44. Tworetzky W, McElhinney D, Reddy V, Brook M, Hanley F, Silverman N. Improved surgical outcome after fetal diagnosis of hypoplastic left heart syndrome. Circulation 2001;103:1269-73.

45. Franklin O, Burch M, Manning N, Sleeman K, Gould $\mathrm{S}$, Archer N. Prenatal diagnosis of coarctation of the aorta improves survival and reduces morbidity. Heart 2002;87:67-9.

46. Soongswang J, Adatia I, Newman C, Smallhorn J, Williams W, Freedom R. Mortality in potential arterial switch candidates with transposition of the great arteries. J Am Coll Cardiology 1998;32:753-7.

47. Mahle W, Forkey H, Wernovsky G, Rhodes LA. Sepsis, septic shock, acute abdomen? The ability of cardiac disease to mimic other medical illness. Pediatr Emerg Care 1996;12:317-24.

48. Grandjean H, Larroque D, Levi S. The performance of routine ultrasonographic screening of pregnancies in the Eurofetus Study. Am J Obstet Gynecol. 1999; 181(2):446-54.

49. Lys F, De Wals P, Borlee-Grimee I, Billiet A, VincotteMols M, Levi S. Evaluation of routine ultrasound examination for the prenatal diagnosis of malformation. Eur J Obstet Gynecol Reprod Biol 1989 $30(2): 101-9$ 